\title{
BMJ Open Association between urbanisation and the risk of hyperuricaemia among Chinese adults: a cross-sectional study from the China Health and Nutrition Survey (CHNS)
}

\author{
Xixi Yu (1D , 1,2,3 Cheng Zhu, ${ }^{1}$ Han Zhang, ${ }^{1,2,3}$ Ziyan Shen, ${ }^{1,2,3}$ Jing Chen, ${ }^{1,2,4}$ \\ Yulu Gu, ${ }^{1,2,4}$ Shiqi Lv, ${ }^{1,2,3}$ Di Zhang, ${ }^{1,2,4}$ Yulin Wang, ${ }^{1,2,4}$ Xiaoqiang Ding, ${ }^{1,2,3,4}$ \\ Xiaoyan Zhang ${ }^{1,2,3,4}$
}

To cite: Yu X, Zhu C, Zhang H, et al. Association between urbanisation and the risk of hyperuricaemia among Chinese adults: a crosssectional study from the China Health and Nutrition Survey (CHNS). BMJ Open 2021;11:e044905. doi:10.1136/ bmjopen-2020-044905

- Prepublication history for this paper is available online. To view these files, please visit the journal online (http://dx.doi. org/10.1136/bmjopen-2020044905).

$X D$ and $X Z$ contributed equally. $\mathrm{XY}$ and $\mathrm{CZ}$ contributed equally.

$\mathrm{XY}$ and $\mathrm{CZ}$ are joint first authors.

Received 17 September 2020 Revised 04 February 2021 Accepted 09 February 2021

Check for updates

(c) Author(s) (or their employer(s)) 2021. Re-use permitted under CC BY-NC. No commercial re-use. See rights and permissions. Published by BMJ.

For numbered affiliations see end of article.

Correspondence to

Dr Xiaoyan Zhang;

zhang.xiaoyan@zs-hospital.sh. cn and

Dr Xiaogiang Ding; dingxiaoqiang2015@hotmail. com

\section{ABSTRACT}

Objective To explore the association between urbanicity and hyperuricaemia (HUA) and whether urbanicity is an independent risk factor for HUA in Chinese adults. Design Data analysis from a cross-sectional survey. Setting and participants 8579 subjects aged 18 years or older were enrolled in the study from the 2009 wave of the China Health and Nutrition Survey to analyse the association between urbanicity and HUA. We divided them into three categories according to urbanisation index (low, medium and highly urbanised groups).

Main outcome measures HUA was defined as serum uric acid $\geq 7 \mathrm{mg} / \mathrm{dL}$ in men and $\geq 6 \mathrm{mg} / \mathrm{dL}$ in women.

Results The prevalence of HUA in low, medium and highly urbanised groups was $12.2 \%, 14.6 \%$ and $19.8 \%$, respectively. The independent factors influencing serum uric acid included age, gender, hypertension, diabetes, chronic kidney disease, drinking, obesity and communitylevel urbanisation index $(\beta=0.016, p<0.001)$. The risk of HUA in the highly urbanised group was significantly higher than that of the low urbanised group (OR 1.771, 95\% Cl 1.545 to $2.029, p<0.001$ ), even after adjusting for other covariates (OR 1.661, 95\% Cl 1.246 to 2.212, $\mathrm{p}=0.001$ ). In a subgroup analysis, we found that age, gender, comorbidity (such as hypertension, diabetes, obesity and chronic kidney disease) and physical activity affected the association between urbanisation and the risk of HUA. Conclusions Our findings suggest that living in highly urbanised areas is linked with higher risk of HUA independent of cardiometabolic and health-related behavioural risk factors, which have been shown to increase along with urbanisation.

\section{INTRODUCTION}

In recent decades, with changes in diet and lifestyle as the economy develops, the prevalence of hyperuricaemia (HUA) has increased rapidly. ${ }^{1}$ In 2014, the prevalence of HUA in Chinese adults was $13.3 \% .^{2}$ HUA is both an independent risk factor for newonset chronic kidney disease $(\mathrm{CKD})^{3}$ as well
Strengths and limitations of this study

- The present study used the 2009 wave of the China Health and Nutrition Survey, which represented $47 \%$ of China's population.

- Regression models were used to explore the association between urbanisation and the risk of hyperuricaemia in Chinese adults.

- The association in women could be impacted by a significant amount of missing data on smoking.

- Even with self-reported history, physical and laboratory examinations, the real prevalence of hypertension and diabetes mellitus might be under-reported.

as CKD progression. ${ }^{45}$ Men and women with HUA are at four and nine times increased risk for end-stage renal disease, respectively. ${ }^{6}$ Furthermore, HUA was reported to increase the risk of diabetes mellitus (DM) ${ }^{7}$ hypertension, ${ }^{8}$ dyslipidaemia ${ }^{9}$ and cardiovascular events, especially sudden cardiac death. ${ }^{1011}$

Urbanicity was confirmed to have an influence on health through nutrition and lifestyle choices, pollution, occupational and traffic hazards, and sanitary conditions such as healthcare access and vaccination coverage. ${ }^{12}{ }^{13}$ Several studies have found that pollution, ${ }^{14}$ drinking, ${ }^{15}$ smoking, ${ }^{16}$ reduced physical activity ${ }^{17} 18$ and fructose intake ${ }^{19}$ were all associated with HUA. Furthermore, some studies have found that renal function was related to urbanicity. ${ }^{21}$ The causes of HUA include increased urate generation, decreased urate excretion or a combination of both factors. Two-thirds of urate is excreted through the kidney into the urine. ${ }^{22}$ Reduced renal function can significantly increase the risk of HUA. ${ }^{23} 24$ 
Few studies have investigated the relationship between urbanicity and HUA. To explore this association, we used data from the China Health and Nutrition Survey (CHNS) and designed a multilevel model to explore whether urbanicity is an independent risk factor for HUA.

\section{MATERIALS AND METHODS}

\section{Sampling and participants}

Sampling in the present study came from the 2009 wave of CHNS. The CHNS is a longitudinal study of nine Chinese provinces (Guizhou, Guangxi, Heilongjiang, Henan, Hubei, Hunan, Liaoning, Jiangsu and Shandong). Nine surveys have been conducted since $1989 .{ }^{25}$ By 2011, the provinces included in the CHNS represented $47 \%$ of China's population according to the 2010 census. The CHNS was designed to provide representation of urban, suburban and rural areas, varying significantly in economic development, public resources, geography and health indicators, and to focus on health during urbanisation and economic change. ${ }^{26}$ We selected a stratified probability sample from the nine provinces using a multistage, random-cluster design. Using this sampling strategy, we selected two cities from each province (one large city, usually the provincial capital, and one small city, usually a lower income city) and four counties (stratified by income: one high-income, one low-income and two middle-income counties). Within cities, we randomly selected two urban and two suburban communities; within counties, we randomly selected one community in the capital city and three rural villages. In each community, we selected 20 households at random and all household members were interviewed. The 2009 wave consisted of 216 communities and included 36 urban neighbourhoods, 36 suburban neighbourhoods, 36 towns and 108 villages. The current study population included 8579 participants aged 18 years and older and the selection procedures are depicted in figure 1.

\section{Urbanicity scale}

Urbanicity was defined using a 12-component index capturing community-level physical, social, cultural and economic environments designed and validated for CHNS. ${ }^{26}$ The following 12 components were included in the development of the urbanisation index: (1) population density; (2) types of economic activity; (3) traditional market; (4) modern markets; (5) transportation and infrastructure; (6) sanitation; (7) communication and media (eg, television, mobile, post and cinema); (8) housing (eg, electricity, indoor tap water and flushing toilets); (9) education; (10) diversity (ie, variation in

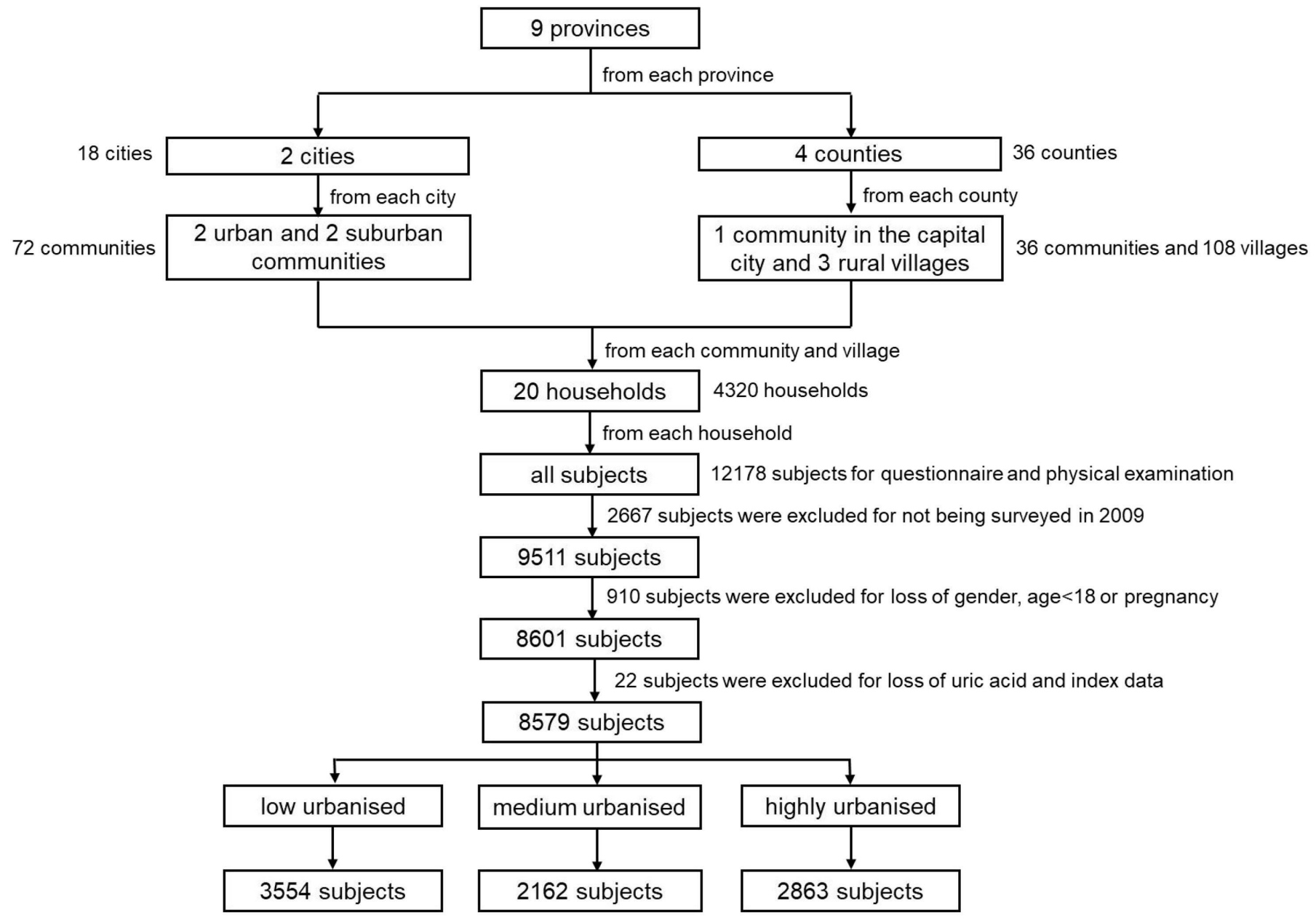

Figure 1 Flow chart of each step of the sample selection method. 
community education level and variation in community income level); (11) health infrastructure; and (12) social services. This scale represents abroad-based factors of modernisation that have potential health effects. Heterogeneity in the presence/absence or number of facilities within the community, access to media or infrastructure, facility characteristics, and the average proportion of individuals and households having a specific education or income level was captured by components. We obtained the variables measuring the proportion of households from the CHNS responses. Using the CHNS community-level survey offered to community officials, we derived the remaining variables as described by JonesSmith and Popkin. ${ }^{26}$ Scoring distributions were variable across components, so the median score in the middle year was designated as half the total points and each of the components was scaled from 0 to $10 .{ }^{27}{ }^{28}$ Each component was then weighted equally in the overall index and added together for an overall maximum possible score of 120 . This scale has been validated for content validity, reliability ( $\alpha=0.85-0.89$ across all study years) and stability across study years $(r=0.90-0.94)$. Since the communitylevel urbanisation indexes in the population we studied ranged from 30.4 to 106.6, all participants were divided into low $(<55.01)$, medium $(\geq 55.01$ and $<82.33)$ and highly ( $\geq 82.33$ ) urbanised groups by their communitylevel urbanisation index tertiles accordingly.

\section{Definition of HUA}

After at least 12 hours of overnight fasting, a blood sample was collected by venipuncture in the morning. Then 4 $\mathrm{mL}$ of the blood sample was collected into a tube with separating gel and was centrifuged $30 \mathrm{~min}$ after collection at $3000 \times g$ for $15 \mathrm{~min}$; the serum sample obtained from the centrifugation was frozen and stored at $-86^{\circ} \mathrm{C}$ for laboratory analysis. Another blood sample $(500 \mu \mathrm{L})$ was collected into a tube with EDTA for routine blood examination. All samples were verified and analysed in a national central laboratory in Beijing (Medical Laboratory Accreditation Certificate ISO 15189:2007) according to strict quality control standards. ${ }^{29}$ Serum uric acid (SUA) concentrations were measured using an enzymatic colourimetric method on a Hitachi 7600 automated analyser (Hitachi, Tokyo, Japan) by determiner reagents (Randox Laboratories, Crumlin, UK). HUA was defined as SUA concentrations $\geq 7 \mathrm{mg} / \mathrm{dL}$ in men and $\geq 6 \mathrm{mg} / \mathrm{dL}$ in women. ${ }^{129-32}$

\section{Assessment of covariates}

Self-reported medical history, including hypertension, DM or high blood sugar, and lifestyle information, such as smoking and drinking, were collected by trained interviewers. Hypertension was defined as either systolic pressure $\geq 140 \mathrm{~mm} \mathrm{Hg}$, diastolic pressure $\geq 90 \mathrm{~mm} \mathrm{Hg}$ or self-reported diagnosis of hypertension. ${ }^{33} \mathrm{DM}$ was defined as either fasting blood glucose $\geq 126 \mathrm{mg} / \mathrm{dL}$ (7.0 $\mathrm{mmol} / \mathrm{L}$ ) or glycosylated haemoglobin $\geq 6.5 \%$ or selfreported diagnosis of DM. ${ }^{34}$ High level of low-density lipoprotein cholesterol (LDL-c) was defined as $\geq 3.12$ mmol/L. ${ }^{35}$ To accurately estimate kidney function, we referred to the Chronic Kidney Disease - Epidemiology Collaboration (CKD-EPI) equation to calculate the estimated glomerular filtration rate $(\mathrm{eGFR})$ : $\mathrm{eGFR}=141 \times$ $\min (\mathrm{SCr} / \kappa, 1)^{\alpha} \times \max (\mathrm{SCr} / \kappa, 1)^{-1.209} \times 0.993^{\mathrm{Age}} \times 1.021$ (if female) $\times 1.159$ (if black), where $\mathrm{SCr}$ is serum creatinine, $\kappa$ is 0.7 for women and 0.9 for men, $\alpha$ is -0.329 for women and -0.411 for men, min indicates the minimum of $\mathrm{SCr} / \mathrm{\kappa}$ or 1 , and max indicates the maximum of $\mathrm{SCr} / \kappa$ or $1 .{ }^{36} \mathrm{CKD}$ was defined as eGFR $<60 \mathrm{~mL} / \mathrm{min} / 1.73 \mathrm{~m}^{2}$ according to the KDIGO (Kidney Disease: Improving Global Outcomes) 2012 Clinical Practice Guideline for the Evaluation and Management of Chronic Kidney Disease ${ }^{36}$ From physical examination, we obtained participants' body weight and height. Body mass index (BMI) was calculated as weight $(\mathrm{kg})$ divided by height squared and was classified into normal or overweight (BMI $<28.0$ $\mathrm{kg} / \mathrm{m}^{2}$ ) and obese $\left(\mathrm{BMI} \geq 28.0 \mathrm{~kg} / \mathrm{m}^{2}\right){ }^{37}$

Physical activity included domestic activity (such as washing clothes, grocery shopping), occupation activity, transportation activity (such as walking or driving to work) and leisure activity (such as kung fu, swimming, playing football $^{38} 39$ and was estimated by metabolic equivalent for task (MET). MET is a unit that estimates the amount of energy used by the body during physical activity, relative to resting metabolism. The unit is standardised so it can apply to people of varying body weight participating in different activities. ${ }^{40}$ Active or inactive group was defined as $\geq 27$ METs/week or $<27$ METs/week according to physical activity level. ${ }^{29}$ Data on alcohol consumption and smoking status of the participants could also be attained from the CHNS and were classified as 'yes' (drink $\geq 1$ per week or smoke currently) or 'no' (drink $<1$ per week or not smoke currently) in our analysis.

\section{Statistical analysis}

Continuous variables were presented as mean $\pm \mathrm{SD}$, while frequencies and percentages were used as categorical variables. One-way analysis of variance test (for continuous variables) and $\chi^{2}$ test (for categorical variables) were used to compare differences in HUA, age, gender, cardiometabolic risk factors (hypertension, DM, high level of LDL-c, obesity, CKD) and health-related behaviours (drinking, smoking, physical activity) among groups, respectively. Additionally, the associations of uric acid with variables were tested using Spearman correlation coefficients in unadjusted and multivariable-adjusted linear regression models.

The method of maximum likelihood by the binary logistics regression model was used to analyse the relationship between the risk of HUA in adulthood and community-level urbanisation exposure. In the multivariable logistic regression model, we adjusted for age, gender, CKD, health-related behaviours and cardiometabolic risk factors. Model 1 was only controlled by age and gender, and model 2 was controlled by factors from model 1 plus cardiometabolic risk factors (obesity, hypertension, 


\begin{tabular}{|c|c|c|c|c|}
\hline Variables & Low urbanised & Medium urbanised & Highly urbanised & $P$ value \\
\hline$n$ & 3554 & 2162 & 2863 & \\
\hline HUA, n (\%) & $435(12.2)$ & $316(14.6)$ & $567(19.8)$ & $<0.001$ \\
\hline Age, mean (SD), year & $50.29(14.76)$ & $50.50(14.94)$ & $52.15(15.37)$ & $<0.001$ \\
\hline Male, n (\%) & $1696(47.7)$ & $1020(47.2)$ & $1327(46.3)$ & 0.549 \\
\hline DM, n (\%) & $296(8.3)$ & $258(11.9)$ & $374(13.1)$ & $<0.001$ \\
\hline High LDL-c, n (\%) & 1209 (34.6) & $1000(46.3)$ & $1261(44.0)$ & $<0.001$ \\
\hline Obesity, n (\%) & $298(8.4)$ & $212(9.8)$ & $312(10.9)$ & $<0.001$ \\
\hline eGFR, mean (SD), mL/min/1.73 $\mathrm{m}^{2}$ & $81.98(16.46)$ & $78.71(16.88)$ & $76.57(16.94)$ & $<0.001$ \\
\hline CKD, n (\%) & $307(8.6)$ & $255(11.8)$ & $437(15.3)$ & $<0.001$ \\
\hline
\end{tabular}

Continuous variables were expressed as mean \pm SD and categorical variables were described as frequencies and percentages.

One-way analysis of variance test (for continuous variables) and $\chi^{2}$ test (for categorical variables) were used to compare differences between different groups.

CKD, chronic kidney disease; DM, diabetes mellitus; eGFR, estimated glomerular filtration rate; HUA, hyperuricemia; LDL-c, low-density lipoprotein cholesterol; MET, metabolic equivalent for task; n, number; SUA, serum uric acid.

DM, high LDL-c, obesity and CKD). For model 3, healthrelated behaviours (smoking, drinking and physical activity) were added for adjustment. All statistical analyses were conducted using the Statistical Package for the Social Sciences V.13.0. Data were demonstrated as OR and $95 \%$ CI. A two-sided $\mathrm{p}$ value $<0.05$ was considered significant.

\section{RESULTS}

\section{Characteristics of participants by tertiles of community-level urbanisation index}

A total of 8579 participants were enrolled in the current study. The basic characteristics of the participants are presented in table 1 . The prevalence of HUA rose with the urbanisation scale (from $12.2 \%$, to $14.6 \%$, to $19.8 \%$; $\mathrm{p}<0.001$ ), as did the trend in mean SUA levels (from 5.02, to 5.16 , to $5.42 ; \mathrm{p}<0.001$ ).

As urbanisation increased, renal function declined dramatically (eGFR reduced from 81.98 , to 78.71 , to 76.57; $\mathrm{p}<0.001)$. In terms of cardiometabolic risk factors, subjects who lived in more urbanised communities were prone to hypertension, DM, high LDL-c and obesity. From the perspective of health-related behaviours, subjects from highly urbanised areas tended to smoke less, drink less and be less physically active compared with those from low urbanised areas.

\section{Risk factors associated with SUA among Chinese adults}

Table 2 shows the results of the univariable and multivariable linear regression analyses between SUA and age, gender, cardiometabolic risk factors and health-related behaviours. The independent factors influencing SUA included age, gender, hypertension, DM, obesity, CKD, drinking and community-level urbanisation index. Men, drinking individuals, individuals with cardiometabolic risk factors (such as hypertension, diabetes, obesity and
CKD) and individuals who lived in a community with higher urbanisation index tended to have higher SUA.

\section{Association of urbanisation and the risk of HUA among Chinese adults}

The association of urbanisation with HUA in Chinese adults is demonstrated in table 3. Compared with low urbanised group, the prevalence of HUA in medium and highly urbanised groups showed significant difference in univariate analysis, as shown in table 1. Even after adjusting for age, gender, cardiometabolic risk factors and health-related behaviours, the highly urbanised group still had higher risk of HUA compared with low urbanised group (OR 1.661, 95\% CI 1.246 to 2.212, $\mathrm{p}=0.001$ ). Furthermore, by subgroup analysis of low and highly urbanised groups, age, gender, comorbidities (such as hypertension, diabetes, obesity and CKD) and physical activity were suggested to affect the association between urbanisation and the risk of HUA (figure 2). Young and middle-aged men living in the community with higher community-level urbanisation index were at higher risk for HUA. Such association also existed in individuals without hypertension, diabetes, obesity or CKD and individuals with less physical activity.

\section{DISCUSSION}

In the current study, we found that individuals living in highly urbanised areas were at higher risk for HUA. The association between urbanicity and HUA remained after adjusting for age, gender and cardiometabolic/healthrelated behavioural risk factors.

Several potential mechanisms could explain the associations between high urbanicity and HUA. High pollution levels are present in highly urbanised areas. ${ }^{41-43}$ Previous studies had shown that air pollution in China 
Table 2 Factors associated with serum uric acid among Chinese adults

\begin{tabular}{|c|c|c|c|c|}
\hline & \multicolumn{2}{|l|}{ Univariable } & \multicolumn{2}{|l|}{ Multivariable } \\
\hline & $\beta$ coefficient & $P$ value & $\beta$ coefficient & Adjusted $\mathrm{p}$ value \\
\hline Age (every 10 years) & 0.019 & $<0.001$ & -0.028 & $<0.001$ \\
\hline Hypertension (yes vs no) & 0.110 & $<0.001$ & 0.047 & $<0.001$ \\
\hline DM (yes vs no) & 0.124 & $<0.001$ & 0.067 & $<0.001$ \\
\hline CKD (yes vs no) & 0.195 & $<0.001$ & 0.198 & $<0.001$ \\
\hline Smoking (yes vs no) & -0.018 & 0.307 & - & - \\
\hline Drinking (yes vs no) & 0.063 & $<0.001$ & 0.037 & 0.001 \\
\hline Physical activity (every 10 METs) & $<0.001$ & 0.700 & - & - \\
\hline
\end{tabular}

The $\beta$ coefficients and $p$ values are from univariable and multivariable linear regression models of natural log-transformed uric acid as the dependent variable. The multivariable model included all covariates.

- , without significance.

CKD, chronic kidney disease; DM, diabetes mellitus; LDL-c, low-density lipoprotein cholesterol; METs, metabolic equivalent for task.

is mainly caused by population aggregation, urbanisation, industrial discharges, outside investment, vehicle exhausts, coal consumption, technological development and straw burning. ${ }^{45}$ Air pollution was reportedly associated with lower eGFR and increased prevalence of CKD, thus increasing the risk of HUA. ${ }^{46}$ Furthermore, previous studies have confirmed that air pollution contained toxic organic agents, including polychlorinated biphenyls, polycyclic aromatic hydrocarbons, perfluorinated alkyl substances and dioxins, which can increase SUA concentrations and the incidence of HUA. ${ }^{47}$ Exposure to greater concentrations of long-term ambient air pollutants has been confirmed to be associated with a higher incidence of HUA. ${ }^{14}$

High urbanicity is accompanied by less physical activity. As found in our study, physical activity declined with increase in urbanicity. In highly urbanised areas, occupational physical activity is less common, as well as transportation and domestic activity, due to the popularity of motorised transportation and household appliances. ${ }^{48}$
Physical exercise is closely associated with SUA, as levels within professional endurance athletes are significantly lower than non-athletes. ${ }^{49}$ After aerobic exercise, SUA increases immediately and then decreases to a level even lower than the pre-exercise level as energy-rich purine phosphates are transiently accumulated and catabolised, followed by a long-lasting depletion. ${ }^{50}$ In our study, inadequate physical activity in individuals living in more urbanised communities increased the risk of HUA.

High urbanicity is associated with decreased kidney function. A previous study which included a large population revealed a higher risk of CKD in a community of higher urbanicity. ${ }^{51}$ As demonstrated in table 1 , the highly urbanised group had the highest prevalence of CKD. Kidney function is responsible for uric acid excretion and SUA, which was consistent with the results in table 2. The SUA level in patients with CKD was higher than those without CKD.

Urbanisation has associations with other noncommunicable diseases, such as diabetes, hypertension,

Table 3 Association of urbanisation and the risk of hyperuricaemia among Chinese adults

\begin{tabular}{|c|c|c|c|}
\hline Variables & Low urbanised & Medium urbanised & Highly urbanised \\
\hline Hyperuricaemia (\%) & 12.2 & 14.6 & 19.8 \\
\hline$P$ value* & & 0.009 & $<0.001$ \\
\hline $\mathrm{P}$ value $†$ & & 0.218 & $<0.001$ \\
\hline OR $(95 \% \mathrm{Cl}) \dagger$ & Ref. & $1.109(0.941$ to 1.308$)$ & 1.548 (1.339 to 1.789$)$ \\
\hline
\end{tabular}

*Model 1 was controlled by age and gender.

†Model 2 was controlled by hypertension, diabetes mellitus, high low-density lipoprotein, obesity and chronic kidney disease based on model 1. ¥Model 3 was controlled by health-related behaviours (smoking, drinking, physical activity) based on model 2 .

Ref, reference. 


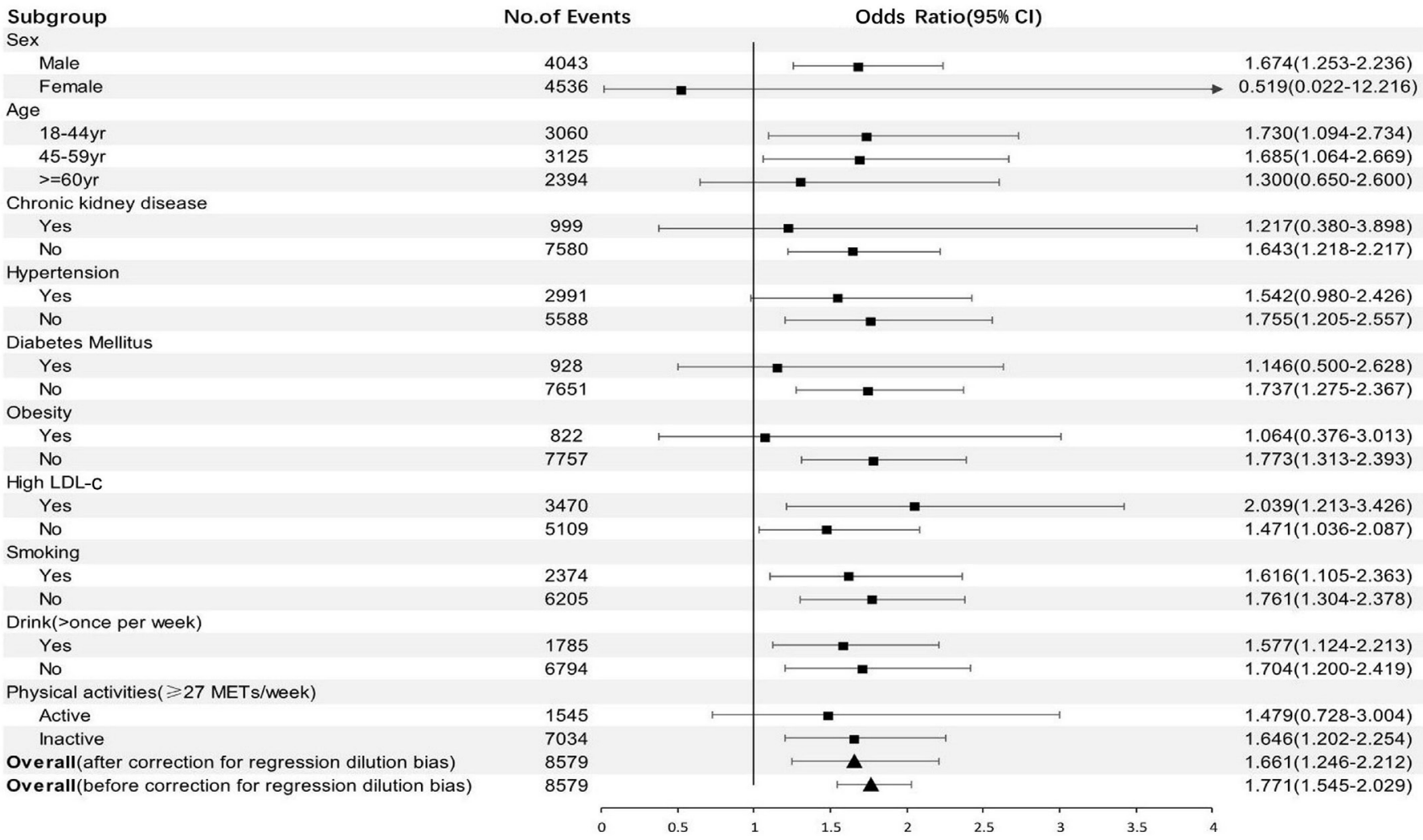

Figure 2 Adjusted OR for hyperuricaemia with high urbanicity according to baseline characteristics. Analyses were adjusted for age, gender, hypertension, diabetes mellitus, high low-density lipoprotein-cholesterol (high LDL-c), obesity, chronic kidney disease, smoking, drinking and physical activity, as appropriate. The square black boxes represent OR and the horizontal lines represent $95 \% \mathrm{Cl}$. The triangle black boxes represent the overall OR and 95\% Cl. METs, metabolic equivalent for task.

high LDL-c, cardiovascular disease, cancer and neuropsychiatric disorders, resulting from changes in human activity, diet and social structures in China. ${ }^{13}$ These diseases can also increase the risk of HUA. ${ }^{171152}$

In addition, we also found that age, gender, comorbidity (such as hypertension, diabetes, obesity and CKD) and physical activity affect the association between urbanisation and the risk of HUA. Young and middle-aged men living in a community with high community-level urbanisation index are at higher risk for HUA. Such association also exists in individuals without hypertension, diabetes, obesity and CKD and in individuals with less physical activity, suggesting that in more urbanised areas individuals without traditional risk factors still have higher risk of HUA. The interaction between urbanicity and hypertension, diabetes, obesity and CKD might conceal the relationship between urbanicity and HUA in these subgroups.

A strength of our study is that the CHNS data we analysed in our survey are widely representative of the entire Chinese mainland. In addition, the innovative grouping and stratifying methods make it clear to distinguish the exact stage in which urbanicity exerts influence on HUA.

Our study also has some limitations. First, elderly individuals in our study tend to have lower SUA. Reduced kidney function, hypertension and diabetes can increase the risk of HUA and are common in elderly individuals. However, elderly individuals usually consume lower purine diets and pay more attention to health-related diets and behaviours compared with younger individuals. Effective diet control can reduce SUA by $1.0-1.2 \mathrm{mg} / \mathrm{dL}$, which can partially explain the relationship between age and SUA. Second, the association between urbanicity and HUA only exists in men after adjusting for cardiometabolic and health-related behavioural risk factors. Women tend to smoke less, drink less and be more inactive compared with men; thus their uric acid level is less influenced by urbanicity. Third, the association in women could be unavoidably affected by a fair amount of missing data on smoking. Fourth, even with self-reported history and physical and laboratory examinations, the real prevalence of hypertension and DM might be under-reported. Finally, the population we analysed was derived from China, and global data are needed to generalise the results.

In conclusion, living in highly urbanised areas is linked with higher risk of HUA independent of health-related behavioural and cardiometabolic risk factors, especially in individuals without traditional HUA risk factors such as hypertension, DM, obesity and CKD.

\section{Author affiliations}

${ }^{1}$ Department of Nephrology, Zhongshan Hospital, Fudan University, Shanghai, China

${ }^{2}$ Shanghai Medical Center of Kidney Disease, Shanghai, China

${ }^{3}$ Shanghai Institute of Kidney and Dialysis, Shanghai, China

${ }^{4}$ Shanghai Key Laboratory of Kidney and Blood Purification, Shanghai, China 
Acknowledgements We would like to acknowledge the team of the National Institute of Nutrition and Food Safety, China Center for Disease Control and Prevention, Carolina Population Center, the University of North Carolina at Chapel Hill, the NIH (R01-HD30880, DK056350, and R01-HD38700) and the Fogarty International Center, NIH for financial support for the CHNS data collection and analysis files from 1989 to 2006 and both parties plus the China-Japan Friendship Hospital, Ministry of Health for support for CHNS 2009 and future surveys. And we also acknowledge all the participants included in this study.

Contributors $X Z$ and $X D$ were coinvestigators and supervisors of the study. $X Y$ carried out the study design, data analysis and writing of the paper. CZ provided the original idea of the paper, the original writing idea of the paper and played a vital role in the revised submission. $\mathrm{HZ}$ and $\mathrm{JC}$ served as scientific advisors and supervised the data analysis. ZS and YG polished the article. SL, DZ and YW collected the data. All authors were involved in writing the paper and had final approval of the submitted and published versions.

Funding This study was supported by research grants from the Ministry of Science and Technology of the People's Republic of China (2016YFC1305500), Shanghai Municipal Health Commission (2017ZZ01015) and the China National Natural Science Foundation (81700646 and 81970637). The funders had no role in study design, data collection and analysis, decision to publish, or preparation of the manuscript.

\section{Competing interests None declared.}

Patient and public involvement Patients and the public had not been involved in the development of the research question or in the design of the study. Patients had received oral and written information about this research; however, they were not involved in the recruitment and conduct of the study. After signing informed consent, they were assessed for eligibility and data collection began. Dissemination of the general results (no personal data) was approved only after the CHNS Review Board qualified the application.

Patient consent for publication Not required.

Ethics approval The CHNS was approved by the Institutional Review Board at the University of North Carolina at Chapel Hill, the China-Japan Friendship Hospital and the Chinese Center for Disease Control and Prevention's National Institute for Nutrition and Health. All subjects gave informed consent for participation. Access to data will be approved by the Institutional Review Board. Analysis of the data presented in this paper was approved by the Ethics Committee of Zhongshan Hospital, Fudan University (B2018-166).

Provenance and peer review Not commissioned; externally peer reviewed.

Data availability statement Data are available in a public, open access repository. The data sets analysed in the current study are available online (https://www.cpc. unc.edu/projects/china/data/datasets/data_downloads/longitudinal).

Open access This is an open access article distributed in accordance with the Creative Commons Attribution Non Commercial (CC BY-NC 4.0) license, which permits others to distribute, remix, adapt, build upon this work non-commercially, and license their derivative works on different terms, provided the original work is properly cited, appropriate credit is given, any changes made indicated, and the use is non-commercial. See: http://creativecommons.org/licenses/by-nc/4.0/.

\section{ORCID iD}

Xixi Yu http://orcid.org/0000-0002-9290-5645

\section{REFERENCES}

1 Zhu Y, Pandya BJ, Choi HK. Prevalence of gout and hyperuricemia in the US general population: the National health and nutrition examination survey 2007-2008. Arthritis Rheum 2011;63:3136-41.

2 Liu R, Han C, Wu D, et al. Prevalence of hyperuricemia and gout in mainland China from 2000 to 2014: a systematic review and metaanalysis. Biomed Res Int 2015;2015:1-12.

3 Li L, Yang C, Zhao Y. Is hyperuricemia an independent risk factor for new-onset chronic kidn ey disease?: a systematic review and metaanalysis based on observatio nal cohort studies. BMC Nephrol:15.

4 Weiner DE, Tighiouart $\mathrm{H}$, Elsayed EF, et al. Uric acid and incident kidney disease in the community. J Am Soc Nephrol 2008;19:1204-11.

5 Kang D-H, Nakagawa T, Feng L. A role for uric acid in the progression of renal disease. J Am Soc Nephrol 2002;13:2888-97.

6 Kunitoshi I. Significance of hyperuricemia as a risk factor for developing ESRD in a screened cohort. American journal of kidney diseases: the official journal of the National Kidney Foundation 2004:4.

7 Sluijs I, Beulens JWJ, van der A DL, et al. Plasma uric acid is associated with increased risk of type 2 diabetes independent of diet and metabolic risk factors. J Nutr 2013;143:80-5.

8 Kansui Y, Matsumura K, Morinaga Y, et al. Impact of serum uric acid on incident hypertension in a worksite population of Japanese men. $J$ Hypertens 2018;36:1499-505.

9 Peng T-C, Wang C-C, Kao T-W, et al. Relationship between hyperuricemia and lipid profiles in US adults. Biomed Res Int 2015;2015:127596.

10 Kleber ME, Delgado G, Grammer TB, et al. Uric acid and cardiovascular events: a Mendelian randomization study. J Am Soc Nephrol 2015;26:2831-8.

11 Feig DI, Kang D-H, Johnson RJ. Uric acid and cardiovascular risk. $N$ Engl J Med 2008;359:1811-21.

12 Vlahov D, Galea S. Urbanization, urbanicity, and health. J Urban Health 2002;79:S1-12.

13 Gong P, Liang S, Carlton EJ. Urbanisation and health in China. The Lancet 2012;379:843-52.

14 Tang Y-X, Bloom MS, Qian Z. Association between ambient air pollution and hyperuricemia in traffic police officers in China: a cohort study. Int J Environ Health Res 2019:1-9.

15 Nakamura K, Sakurai M, Miura K. Alcohol intake and the risk of hyperuricaemia: a 6-year prospective study in Japanese men. Nutrition, Metabolism and Cardiovascular Diseases 2012;22:989-96.

16 Yang T, Zhang Y, Wei J, et al. Relationship between cigarette smoking and hyperuricemia in middle-aged and elderly population: a cross-sectional study. Rheumatol Int 2017;37:131-6.

17 Liu L, Lou S, Xu K, et al. Relationship between lifestyle choices and hyperuricemia in Chinese men and women. Clin Rheumatol 2013;32:233-9.

18 Veronese N, Stubbs B, Trevisan C, et al. Results of an observational cohort study of hyperuricemia as a predictor of poor physical performance in the elderly. Arthritis Care Res 2017;69:1238-44.

19 Cicero AFG, Fogacci F, Desideri G. Arterial stiffness, sugarsweetened beverages and fruits intake in a rural population sample: data from the Brisighella heart study. Nutrients 2019:11.

20 Wang S, Chen R, Liu Q, et al. Prevalence, awareness and treatment of chronic kidney disease among middle-aged and elderly: the China health and retirement longitudinal study. Nephrology 2015;20:474-84.

21 Kaze FF, Meto DT, Halle M-P, et al. Prevalence and determinants of chronic kidney disease in rural and urban Cameroonians: a crosssectional study. BMC Nephrol 2015;16:117.

22 Sorensen LB. Role of the intestinal tract in the elimination of uric acid.

23 Krishnan E. Reduced glomerular function and prevalence of gout: NHANES 2009:10.

24 Madero M, Sarnak MJ, Wang X, et al. Uric acid and long-term outcomes in CKD. Am J Kidney Dis 2009;53:796-803.

25 Popkin BM, Du S, Zhai F, et al. Cohort Profile: The China Health and Nutrition Survey--monitoring and understanding socioeconomic and health change in China, 1989-2011. Int J Epidemiol 2010;39:1435-40.

26 Jones-Smith JC, Popkin BM. Understanding community context and adult health changes in China: development of an urbanicity scale. Soc Sci Med 2010;71:1436-46.

27 DeVellis R. Scale development: theory and applications 1991.

28 Dahly DL, Adair LS. Quantifying the urban environment: a scale measure of urbanicity outperforms the urban-rural dichotomy. Soc Sci Med 2007;64:64.

29 Thompson AL, Houck KM, Adair L, et al. Multilevel examination of the association of urbanization with inflammation in Chinese adults. Health Place 2014;28:177-86.

30 Becker MA, Schumacher HR, Wortmann RL, et al. Febuxostat compared with allopurinol in patients with hyperuricemia and gout. $N$ Engl J Med 2005;353:2450-61.

31 Zhang W, Doherty M, Pascual E, et al. EULAR evidence based recommendations for gout. Part I: diagnosis. Report of a task force of the standing Committee for international clinical studies including therapeutics (ESCISIT). Ann Rheum Dis 2006;65:1301.

32 Perez-Ruiz F, Lioté F. Lowering serum uric acid levels: what is the optimal target for improving clinical outcomes in gout? Arthritis Rheum 2007;57:1324-8.

33 Williams B GM. W S, et al. [2018 ESC/ESH Guidelines for the management of arterial hypertension]. Eur Heart J 2019;77:71.

34 5. Lifestyle Management: <em>Standards of Medical Care in Diabetes-2019</em\&gt. Diabetes Care 2019;42:S46. 
35 Mach F, Baigent C, Catapano A. 2019 ESC/EAS guidelines for the management of dyslipidaemias: lipid modification to reduce cardiovascular risk. Atherosclerosis 2019.

36 Andrassy KM. Comments on 'KDIGO 2012 Clinical Practice Guideline for the Evaluation and Management of Chronic Kidney Disease'. Kidney Int 2013;84:622-3.

37 Zhou B-F. Effect of body mass index on all-cause mortality and incidence of cardiovascular diseases--report for meta-analysis of prospective studies open optimal cut-off points of body mass index in Chinese adults. Biomed Environ Sci 2002;15:245.

38 Ainsworth BE, Haskell WL, Whitt MC, et al. Compendium of physical activities: an update of activity codes and Met intensities. Med Sci Sports Exerc 2000;32:S498-504.

$39 \mathrm{Ng} \mathrm{SW}$, Norton EC, Popkin BM. Why have physical activity levels declined among Chinese adults? findings from the 1991-2006 China health and nutrition surveys. Soc Sci Med 2009;68:1305-14.

40 Sallis JF, Haskell WL, Wood PD, et al. Physical activity assessment methodology in the Five-City project. Am J Epidemiol 1985;121:91-106.

$41 \mathrm{Xu} \mathrm{W}$, Sun J, Liu Y, et al. Spatiotemporal variation and socioeconomic drivers of air pollution in China during 2005-2016. $J$ Environ Manage 2019;245:66-75.

42 Zhang L, Liu L, Zhao Y. Source Attribution of particulate matter pollution over North China with the adjoint method. Environmental Research Letters 2015;10:084011.

43 Han L, Zhou W, Pickett STA, et al. An optimum city size? The scaling relationship for urban population and fine particulate (PM(2.5)) concentration. Environ Pollut 2016;208:96-101.
44 Luo K, Li G, Fang C, et al. $\mathrm{PM}_{25}$ mitigation in China: Socioeconomic determinants of concentrations and differential control policies. $J$ Environ Manage 2018;213:47-55.

$45 \mathrm{Xu} \mathrm{B}$, Lin B. What cause large regional differences in PM2.5 pollutions in China? Evidence from quantile regression model. $J$ Clean Prod 2018;174:447-61.

46 Wang W, Wu C, Mu Z, et al. Effect of ambient air pollution exposure on renal dysfunction among hospitalized patients in Shanghai, China. Public Health 2020;181:196-201.

47 Chang J-W, Ou H-Y, Chen H-L. Hyperuricemia after exposure to polychlorinated dibenzo-p-dioxins and dibenzofurans near a highly contaminated area. Epidemiology 2013;24:582-9.

48 Monda KL, Gordon-Larsen P, Stevens J, et al. China's transition: the effect of rapid urbanization on adult occupational physical activity. Soc Sci Med 2007:64:858-70.

49 Lippi G, Brocco G, Franchini M, et al. Comparison of serum creatinine, uric acid, albumin and glucose in male professional endurance athletes compared with healthy controls. Clin Chem Lab Med 2004;42:644-7.

50 Shi M, Wang X, Yamanaka T, et al. Effects of anaerobic exercise and aerobic exercise on biomarkers of oxidative stress. Environ Health Prev Med 2007;12:202-8.

51 Inoue $\mathrm{Y}$, Howard AG, Thompson AL, et al. The association between urbanization and reduced renal function: findings from the China health and nutrition survey. BMC Nephrol 2017;18:160.

52 Ali N, Mahmood S, Islam F, et al. Relationship between serum uric acid and hypertension: a cross-sectional study in Bangladeshi adults. Sci Rep 2019;9:9061. 